\title{
Ações de Fisioterapia voltadas à saúde da criança em uma escola de ensino fundamental em Maceió, Alagoas
}

\section{Physiotherapy actions directed for children's health at a primary school in Maceió, Alagoas}

\author{
Clarissa Cotrim dos Anjos \\ Fisioterapeuta, Orientadora, graduada pela Universidade Estadual de Ciências da Saúde de Alagoas (Uncisal), especialista em Ciências da Saúde e \\ Neurofuncional, pelo Centro Universitário Cesmac e pela Uncisal \\ clacotrimanjos@gmail.com \\ Shirley da Silva Santos \\ Graduanda em Fisioterapia pela Universidade Estadual de Ciências da Saúde de Alagoas (Uncisal) \\ shyr_1@ hotmail.com \\ Rayane da Costa Silva \\ Graduanda em Fisioterapia pela Universidade Estadual de Ciências da Saúde de Alagoas (Uncisal) rayanefisio@ outlook.com \\ Raysa da Costa Silva \\ Graduanda em Fisioterapia pela Universidade Estadual de Ciências da Saúde de Alagoas (Uncisal) \\ raysa-costa@outlook.com \\ Raiany Azevedo dos Santos Gomes \\ Graduanda em Fisioterapia pela Universidade Estadual de Ciências da Saúde de Alagoas (Uncisal) \\ raianyazevedo-laje@hotmail.com
}

\begin{abstract}
Resumo: A linha de cuidado da saúde da criança é uma prioridade que tem como eixo estruturante o acompanhamento do crescimento e desenvolvimento. A assistência baseia-se na promoção da saúde, na prevenção, no diagnóstico precoce e na recuperação dos agravos à saúde. Este trabalho teve como objetivo relatar a experiência no acompanhamento de atividades desenvolvidas em uma escola na cidade de Maceió, em Alagoas. Trata-se de um estudo descritivo, do tipo relato de experiência, acerca de ações voltadas para a saúde da criança, envolvendo temáticas sobre acompanhamento do crescimento e desenvolvimento, imunização e atividades de educação em saúde. Foram realizadas as medidas antropométricas de cada criança. Das 24 crianças medidas e pesadas, verificou-se que oito estavam com sobrepeso e três com obesidade. Ao avaliar o preenchimento das cadernetas de saúde da criança, apenas nove responsáveis atenderam às solicitações e apenas uma estava com todas as informações preenchidas adequadamente. As crianças compreendidas na faixa etária de 0 a 6 anos foram avaliadas em relação ao desenvolvimento por meio da escala de triagem de Denver II. Esta foi aplicada em 21 crianças, sendo identificado atraso no desenvolvimento de três. Diante deste resultado, foram realizadas intervenções fisioterapêuticas na própria escola e, após três meses de acompanhamento, as crianças apresentaram uma melhora significativa em todos os domínios da escala.
\end{abstract}

Palavras-chave: Crianças Pré-escolares; Desempenho Psicomotor; Saúde Pública

\begin{abstract}
The child health care line is a priority whose structuring axis is the monitoring of growth and development and assistance is based on the promotion of health, prevention, early diagnosis and recovery of health problems. This work had the objective of reporting the experience in the follow up of activities developed in a school in the city of Maceió, Alagoas. This is a descriptive study, a type of experience report about actions directed at children's health, involving the following topics: growth and development monitoring, immunization and health education activities. The anthropometric measurements of each child were performed. Of the 24 children, measured and weighed, 8 were overweight and 3 were obese. When evaluating the completion of the children's health records, only 9 caregivers responded to the requests and only one was adequately filled. Children aged 0-6 years were evaluated for development using the Denver II screening scale. This was applied in 21 children, with a delay in the development of three. In view of this result, physiotherapeutic interventions were performed in the school itself and, after three months of follow-up, showed a significant improvement in all domains of the scale.
\end{abstract}

Key words: Child Preschool; Psychomotor Performance; Public Health 


\section{INTRODUÇÃO}

No Brasil, o Sistema Único de Saúde (SUS) determina que, em cada nível de atenção - primário secundário e terciário, sejam organizadas ações para prevenir agravos, reduzir danos e promover mais qualidade de vida para os cidadãos. Embora cada nível possua sua especificidade no contexto da saúde da criança, recomendam-se atendimentos com enfoque educativo que incentivem a aquisição de hábitos de vida saudáveis ao longo do desenvolvimento (CRISTO; ARAÚJO, 2015).

A Linha de Cuidado é uma estratégia de ação que busca a integralidade da atenção, um dos princípios do Sistema Único de Saúde (SUS), articulando a produção do cuidado desde a atenção primária até os serviços de maior complexidade, no contexto de consolidação das Redes de Atenção à Saúde. Essa Linha de Cuidado tem atenção especial na Primeira Infância, pois a ciência mostra que os primeiros anos de vida das crianças são fundamentais para estabelecer os alicerces de suas aquisições futuras (SÃO PAULO, 2012).

As linhas de cuidados por ciclo de vida englobam: a Saúde da Criança e do Adolescente, a Saúde do Adulto e a Saúde do Idoso. Estas linhas são organizadas de acordo com as necessidades de cada ciclo da vida ou cada paciente, visando à integralidade do cuidado e dos profissionais. (GRABOIS, 2011). No nosso caso, o foco é a criança e os cuidados devem ser direcionados dentro da família e da sociedade.

A atenção integral à saúde da criança organiza-se em três eixos principais, que compreendem ações que vão da anticoncepção à concepção, da atenção ao parto e ao puerpério, passando pelos cuidados com o recém-nascido (acompanhamento do crescimento e desenvolvimento, triagem neonatal, aleitamento materno, doenças prevalentes da infância e saúde coletiva em instituições de educação infantil). As linhas estratégicas de intervenção da atenção à saúde da criança explicitam o conceito de integralidade por meio da oferta de ações educativas, promocionais, preventivas, de diagnóstico e de recuperação da saúde (ERDMANN; SOUSA, 2009).

A linha de cuidado da saúde da criança é uma prioridade que tem como eixo estruturante o acompanhamento do crescimento e desenvolvimento e a assistência, baseando-se na promoção da saúde, prevenção, diagnóstico precoce e recuperação dos agravos à saúde. Um dos instrumentos já utilizados para esse acompanhamento é a Caderneta de Saúde da Criança, distribuída universalmente pelo Ministério da Saúde para todos os nascidos vivos das maternidades públicas e privadas, em cumprimento ao estabelecido na Portaria $\mathrm{n}^{\circ}$ 1.058, de 4 de julho de 2005; para que profissionais da Saúde e familiares registrem pontos importantes do crescimento e do cotidiano infantil, imunização, peso, altura, aquisições do desenvolvimento, alimentação, brincadeiras preferidas, dentre outras informações (BRASIL, 2004).

As principais linhas de cuidado da saúde da criança abordam: atenção humanizada e qualificada à gestante e ao recém-nascido; combate à desnutrição e anemias carenciais; alimentação saudável e prevenção do sobrepeso e obesidade infantil; incentivo e qualificação do acompanhamento e desenvolvimento; atenção à criança portadora de deficiência; prevenção de acidentes, maustratos/violência e trabalho infantil; atenção à saúde mental; atenção à saúde bucal; atenção às doenças prevalentes; imunização; triagem neonatal: teste do pezinho; e incentivo ao aleitamento (VOLTA REDONDA, 2015).

Na década de 1980, em uma ação coordenada entre o governo federal, as secretarias estaduais e municipais de saúde e o Ministério da Saúde, foi elaborado o Programa de Atenção Integral à Saúde da Criança (Paisc), por meio de cinco ações básicas: aleitamento materno, orientação alimentar para o desmame, imunização, e acompanhamento do crescimento e do desenvolvimento. Com este programa, o Ministério da Saúde marcou uma diretriz política para a expansão e a consolidação da rede de serviços básicos, utilizando a estratégia da assistência integral. $\mathrm{O}$ acompanhamento do crescimento e do desenvolvimento foi $\mathrm{o}$ eixo integrador das práticas assistenciais à criança por meio da sistematização de retornos ao serviço de saúde (ERDMANN; SOUSA, 2009).

Desta forma, o Ministério da Saúde quando apresentou esta proposta, visou deslocar o enfoque de uma assistência médico-curativista, centrada na atenção à demanda espontânea às crianças doentes para garantir a integralidade na assistência prestada pelos serviços de saúde (NOVACZYK et al., 2008).

Diante deste contexto, a aproximação entre a fisioterapia e o nível primário da saúde apresenta-se como alternativa capaz de fortalecer a atenção básica, aumentando a resolutividade do sistema e contribuindo para a garantia da integralidade na assistência. A Fisioterapia, embora historicamente tenha se mantido no nível da reabilitação, possui competências e habilidades suficientes para a atuação em outros níveis (JÚNIOR, 2010).

Por acreditar que as linhas do cuidado da criança são de fundamental importância, pois visam acompanhar o desenvolvimento e o crescimento infantil nos primeiros anos de vida e instituir ações educativas, que promovam a aprendizagem dos indivíduos, diagnósticos precoces e recuperar os agravos à saúde, justifica-se o relato da experiência vivenciada pelos discentes do Curso de Fisioterapia no desenvolvimento desta abordagem centrada na atenção primária com foco na integralidade, mudando a visão da saúde curativista e valorizando a nova prática do modelo assistencial.

Este trabalho tem como objetivo relatar a experiência no acompanhamento de atividades desenvolvidas em uma escola com ações voltadas para a saúde da criança na cidade de Maceió, Alagoas.

\section{METODOLOGIA}

Este estudo é caracterizado como descritivo, do tipo relato de experiência acerca de ações voltadas para a saúde da criança, envolvendo temáticas sobre: acompanhamento do crescimento e desenvolvimento, imunização e atividades de educação em saúde/promoção da saúde.

A sistematização da experiência é definida como aquela interpretação crítica de uma ou várias experiências que, a partir de seu ordenamento e reconstrução, descobre 
ou explicita a lógica do processo vivido, os fatores que intervieram no dito processo, como se relacionaram entre si e por que o fizeram desse modo (PEREIRA et al., 2015).

As ações foram desenvolvidas em uma escola na cidade de Maceió (AL), que assiste à 24 crianças na faixa etária de 2 a 8 anos.

Para a fundamentação teórica do estudo, recorreu-se à produção científica nesta área, em artigos publicados em periódicos nacionais, disponíveis na base de dados da Scielo e Lilacs e em manuais/diretrizes do Ministério da Saúde.

Para iniciar as atividades, foi realizado um planejamento das ações que seriam desenvolvidas pelos acadêmicos de Fisioterapia, por meio de reuniões de planejamento $\mathrm{O}$ planejamento das ações foi semestral, ocorrendo no período de fevereiro a junho de 2016. Foram realizados 18 encontros com duração média de duas horas. Todas as atividades foram desenvolvidas nas instalações da própria escola - quando necessário algum material para o desenvolvimento das ações, os mesmos eram deslocados da Clínica Escola de Fisioterapia, e depois devolvidos.

Foram realizadas as medidas antropométricas de peso e estatura de cada criança e colocadas no gráfico constante na caderneta da criança. Destaca-se que a caderneta foi solicitada aos responsáveis para conferência da imunização das mesmas. Posteriormente, as crianças, compreendidas na faixa etária de 0 a 6 anos, foram avaliadas do ponto de vista do desenvolvimento por meio da escala de triagem de Denver II.

\section{RESULTADOS E DISCUSSÃO}

Foi informada a matrícula de 24 crianças na escola, sendo 13 do gênero masculino e 11 do gênero feminino, compreendidas na faixa etária de 0 a 8 anos e a presença de duas professoras.

Foi realizado um cadastramento de todas as crianças e professores, e foram estabelecidas as ações a serem desenvolvidas, bem como a realização de um diagnóstico situacional.

Observou-se que uma criança apresentava dificuldade de entendimento acerca da família. A compreensão sobre a importância da família se faz necessária para o desenvolvimento integral do ser, visto que a família pode ser considerada o sistema que mais influencia diretamente no desenvolvimento da criança. Segundo Silva e Nancy et al. (2008) a família é concebida como o primeiro sistema no qual um padrão de atividades, papéis e relações interpessoais são vivenciados pela pessoa em desenvolvimento e cujas trocas dão base para o estudo do desenvolvimento do indivíduo.

Desta forma, no segundo contato com as crianças, foi realizada uma roda de conversa sobre os "tipos de família". Neste momento, os acadêmicos e a professora puderam ter um real conhecimento da situação familiar de cada criança. Com isso, criou-se um espaço no qual as crianças expressaram o que sabiam e os temas que tinham dúvidas, abordando de forma real suas necessidades, que poderiam gerar impacto no desenvolvimento. Assim, a partir deste momento, os temas das atividades de educação em saúde foram definidos. Foram eles: Práticas de Hábitos Saudáveis; Importância da Vacinação; Incentivo à Saúde
Bucal e Higiene Corporal; Combate ao Aedes Aegypti e Acompanhamento do Crescimento e Desenvolvimento destas Crianças.

Na infância, por meio da avaliação de parâmetros de crescimento e desenvolvimento, é possível detectar inúmeras alterações que podem ocorrer durante a vida de um indivíduo. Desta forma, é necessário que os profissionais sigam as diretrizes do Ministério da Saúde e percebam que o acompanhamento do crescimento e desenvolvimento na atenção básica é importante para a prevenção de doenças nas fases subsequentes da vida de cada criança. Além disso, este acompanhamento serve como norteador para o desenvolvimento de estratégias de promoção da saúde, para identificar diagnósticos precoces e traçar metas visando a recuperação de agravos (LIMA et al., 2009).

As medidas antropométricas foram realizas em 24 crianças, sendo 13 do sexo masculino e 11 do feminino. Os resultados obtidos e as suas intepretações, fazendo uso do índice de massa corporal (IMC), estão descritos na tabela 01 .

Tabela 01. Medidas antropométricas de peso e estatura de crianças em escola na cidade de Maceió, Alagoas

\begin{tabular}{|c|c|c|c|}
\hline MEDIDA & MENINOS & MENINAS & TOTAL \\
\hline $\begin{array}{l}\text { ANTRPRO } \\
\text { MÉTRICA }\end{array}$ & & & $\mathrm{N} \quad \%$ \\
\hline \multicolumn{4}{|l|}{ PESO } \\
\hline Adequado & 10 & 9 & $1979,16 \%$ \\
\hline Elevado & 3 & 2 & $5 \quad 20,83 \%$ \\
\hline \multicolumn{4}{|l|}{ ALTURA } \\
\hline Adequado & 11 & 10 & $2187,5 \%$ \\
\hline Inadequado & 2 & 1 & $3 \quad 12,5 \%$ \\
\hline \multicolumn{4}{|l|}{ IMC } \\
\hline Adequado & 7 & 6 & $1354,16 \%$ \\
\hline Sobrepeso & 5 & 3 & $8 \quad 33,33 \%$ \\
\hline Obesidade & 1 & 2 & $12,5 \%$ \\
\hline
\end{tabular}

Legenda: $\mathrm{N}$ - número absoluto e $\%$ - percentual

Nos últimos 30 anos, a incidência de obesidade em crianças cresceu de menos de 5\% para aproximadamente $20 \%$ nos Estados Unidos. No Brasil, estima-se que 33\% das crianças entre 5 e 9 anos de idade estejam acima do peso, sendo que destas $14,3 \%$ são consideradas obesas, conforme pesquisa de orçamentos familiares em 2008 e 2009.

Crianças com obesidade e sobrepeso apresentam maior incidência de fatores de risco para o desenvolvimento de doença arterial coronariana, devido a alterações metabólicas que se manifestam, mais frequentemente detectada na idade adulta, como alterações dos lipídeos séricos, metabolismo da glicose, hipertensão arterial e anormalidades da coagulação. As graves consequências da obesidade, as dificuldades para o seu controle, o aumento expressivo de sua prevalência nas diversas faixas etárias e o alto custo gerado para a sociedade, fazem dela um relevante problema de saúde pública que precisa ser abordado desde as idades mais precoces (PANAZZOLO et al., 2014). Isso justifica a importância de conscientizar as crianças sobre a prática de hábitos saudáveis, como a prática de atividades físicas que devem ser implantadas dentro da própria escola e a reeducação alimentar. 
Foi observado que o lanche das crianças era de responsabilidade de seus pais e, geralmente, se tratavam de biscoitos recheados, refrigerantes e salgados.

Após a realização da atividade em saúde sobre a necessidade de uma alimentação saudável, observou-se uma mudança nos lanches das crianças, bem como uma maior conscientização das mesmas.

As crianças compreendidas na faixa etária de 0 a 6 anos foram avaliadas com Escala de Triagem de Denver II. O teste pode ser aplicado por vários profissionais da saúde em crianças de 0 a 6 anos, classificando-as dicotomicamente em risco ou normal. O teste é composto por 125 itens distribuídos na avaliação de quatro áreas distintas do desenvolvimento neuropsicomotor motricidade ampla, motricidade fina-adaptativa, comportamento pessoal-social e linguagem. Estes itens são registrados por meio da observação direta da criança e, para alguns deles, solicita-se que a mãe informe se o filho realiza ou não determinada tarefa. O teste Denver II apresenta bons índices de validade e confiabilidade $(0,99$ interobservador e 0,9 em teste reteste) e, portanto, largamente utilizado tanto em pesquisas quanto na prática clínica (SILVA et al., 2011).

Das 21 crianças avaliadas, três apresentaram falhas em mais de três itens na escala, dentre os 4 domínios, em especial no domínio motor grosseiro e motor finoadaptativo. Destaca-se que duas destas crianças eram meninos de 3 anos, que nasceram gemelares $e$ prematuros, mas nunca haviam sido encaminhados para acompanhamento, e um menino de 2 anos.

Desta maneira, os acadêmicos supervisionados pela professora realizaram o acompanhamento do desenvolvimento das crianças nas dependências da escola. As crianças, após três meses de acompanhamento, apresentaram uma melhora significativa em todos os domínios da escala.

De acordo com o Ministério da Saúde, os profissionais de saúde deverão estar aptos a reconhecerem seus aspectos mais relevantes e identificar aquelas crianças que devem ser encaminhadas para tratamento especializado. Entre estes, o fisioterapeuta capacitado para avaliação do desenvolvimento infantil tem potencial para realizar as avaliações de forma precoce, podendo desempenhar um papel importante nas ações primárias de saúde dentro das comunidades. É consensual que, quanto mais precoces forem o diagnóstico de atraso no desenvolvimento e as ações de intervenção, menor será o impacto de danos futuros (AMORIM et al., 2009).

Após realizar as medidas antropométricas e a avaliação do desenvolvimento das crianças, os dados foram inseridos nas cadernetas de saúde. Para tanto, foi enviado aos pais/responsáveis um bilhete solicitando o envio das cadernetas para análise. Entretanto, de 24 cadernetas possíveis de serem analisadas, apenas 9 (37,5\% do total) responderam à solicitação, mesmo realizando uma conscientização das crianças sobre o envio da mesma.

Das cadernetas que foram enviadas para análise, verificou-se uma falta de preenchimento em campos básicos e de extrema importância para o acompanhamento da saúde da criança, como pode ser observado na tabela 02 .
Tabela 02. Análise das cadernetas de crianças em escola na cidade de Maceió, Alagoas.

\begin{tabular}{|c|c|c|c|c|c|c|}
\hline \multirow{2}{*}{$\begin{array}{l}\text { Informações na } \\
\text { caderneta da } \\
\text { saúde da criança }\end{array}$} & \multicolumn{2}{|c|}{ Completo } & \multicolumn{2}{|c|}{ Incompleto } & \multicolumn{2}{|c|}{ Ausente } \\
\hline & $\mathrm{N}$ & $\%$ & $\mathrm{~N}$ & $\%$ & $\mathrm{~N}$ & $\%$ \\
\hline $\begin{array}{c}\text { Dados sobre a } \\
\text { gravidez }\end{array}$ & - & - & 5 & $55,55 \%$ & 4 & $44,44 \%$ \\
\hline $\begin{array}{l}\text { Dados sobre o } \\
\text { parto e pós-parto }\end{array}$ & 3 & $33,33 \%$ & 2 & $22,22 \%$ & 4 & $44,44 \%$ \\
\hline $\begin{array}{l}\text { Dados sobre o } \\
\text { nascimento da } \\
\text { criança }\end{array}$ & 1 & $11,11 \%$ & 6 & $66,66 \%$ & 2 & $22,22 \%$ \\
\hline $\begin{array}{c}\text { Dados sobres } \\
\text { exames /triagem } \\
\text { neonatal }\end{array}$ & - & & 2 & $22,22 \%$ & 7 & $77,77 \%$ \\
\hline $\begin{array}{c}\text { Registro de } \\
\text { medidas } \\
\text { antropométricas } \\
\text { mensal }\end{array}$ & 1 & $11,11 \%$ & 7 & $77,77 \%$ & 1 & $11,11 \%$ \\
\hline $\begin{array}{c}\text { Registro de } \\
\text { alcance dos } \\
\text { marcos do } \\
\text { desenvolvimento }\end{array}$ & - & & 1 & $11,11 \%$ & 8 & $88,88 \%$ \\
\hline
\end{tabular}

Legenda: $\mathrm{N}$ - número absoluto e \% - percentual

No desenvolvimento das ações voltadas para a vigilância à saúde, a Caderneta de Saúde da Criança (CSC) apresenta-se como instrumento essencial de vigilância, por ser o documento onde são registrados os dados e eventos mais significativos para a saúde infantil, por possibilitar o diálogo entre a família e os diversos profissionais que atendem a criança e, especialmente, por pertencer à criança e à família e com elas transitar pelos diferentes serviços e níveis de atenção demandados no exercício do cuidado com a saúde (ALVES et al., 2009).

Além do registro de vacinas, comporta o registro da história obstétrica e neonatal; indicadores do crescimento e desenvolvimento; aspectos importantes da alimentação, como aleitamento materno, uso de sulfato ferroso e vitamina $\mathrm{A}$; dados sobre a saúde bucal, auditiva e visual, incluindo intercorrências clínicas; e orientações para a promoção da saúde e para a prevenção de agravos, como acidentes e violência doméstica (ROCHA, 2012).

As atividades de educação em saúde foram utilizadas na abordagem com os participantes por meio de uma metodologia ativa, buscando despertar o interesse e o diálogo entre as crianças acerca dos aspectos relacionados ao cuidado da saúde da criança, com momentos de construção, reconstrução e desconstrução de conceitos e ideias, estimulando e influenciando o processo do cuidar deste grupo populacional (PEREIRA et al., 2015).

Foram realizadas quatro atividades de educação em saúde com as crianças com as seguintes temáticas: entendendo os tipos de família; imunização; alimentação saudável; higiene corporal e saúde bucal; e cuidados com o mosquito Aedes aegypti (Tabela 03).

Tabela 03. Atividades de educação em saúde realizada com crianças em escola na cidade de Maceió, Alagoas .

\begin{tabular}{lc}
\hline \multicolumn{1}{c}{ Atividade } & Metodologia adotada \\
\hline Perdendo o medo de vacina & Dramatização \\
& Dramatização e roda de \\
Alimentação Saudável & conversa \\
Higiene Corporal e & Dramatização, \\
Saúde Bucal & Demonstração e \\
& musicalização. \\
Mosquito mal & Dramatização \\
\hline
\end{tabular}


A escola é espaço de grande relevância para promoção da saúde, principalmente quando exerce papel fundamental na formação do cidadão crítico, estimulando a autonomia, o exercício de direitos e deveres, o controle das condições de saúde e qualidade de vida, com opção por atitudes mais saudáveis (BRASIL, 2009).

A utilização de metodologias ativas aumenta o potencial da ação educativa ao produzir melhorias no processo saúde/doença, estimulando a construção e o redimensionamento de conceitos e significados sobre os fatores que interferem na própria saúde (ARAÚJO et al., 2013).

Assim, as atividades de educação em saúde foram todas realizadas de forma lúdica e dinâmica, de modo que a criança fizesse parte de todo o processo. Para tanto, foram utilizados recursos como a dramatização, rodas de conversas, demonstração, musicalização e uso de cartazes para ilustrar.

Observou-se uma grande aceitação e interesse por parte das crianças, e uma mudança de hábitos nas mesmas.

Sabe-se que os comportamentos aprendidos em uma fase precoce da vida, tendem a perpetuar-se ao longo da vida adulta. Isso é possível graças a melhor plasticidade neural que a criança apresenta nessa idade, o que reforça a importância destes temas, tratados em nossa experiência, serem abordados nos primeiros anos de vida, proporcionando, assim, uma melhor fixação e um melhor aprendizado.

\section{CONCLUSÕES}

Percebe-se a importância de se realizar ações de Fisioterapia voltadas à saúde da criança, com o enfoque no crescimento e desenvolvimento, imunização e atividades de educação em saúde/promoção da saúde.

\section{REFERÊNCIAS}

ALVES, C. R. L.; LASMAR, L. M. de L. B. F.; GOULART, L. M. H. F.; ALVIM C. G.; MACIEL, G. V. R.; VIANA, M. R. de A.; COLOSIMO, E. A.; CARMO, G. A. A. do; COSTA, J. G. D. da; MAGALHÃES, M. E. N.; MENDONÇA, M. L. de; BEIRÃO, M. M. do V.; MOULIN, Z. S. Qualidade do preenchimento da Caderneta de Saúde da Criança e fatores associados. Cad. Saúde Pública, Rio de Janeiro, v. 25, n. 3, p. 583-595, Mar. 2009.

AMORIM, R. C. A.; LAURENTINO, G. E. C.; BARROS, K. M. F. T.; FERREIRA, A. L. P. R.; FILHO, A. G. M.; RAPOSO, M. C. F. Programa de Saúde da Família: proposta para identificação de fatores de risco para o desenvolvimento neuropsicomotor. Revista Brasileira de Fisioterapia, São Carlos, Maio. 2009.

ARAÚJO, M. G. de; ARAÚJO, M. G. de; CASSIANO, A. do N.; HOLANDA, C. S. M. de; MOREIRA, P. V. S. de Q.; GIOVANNINI, P. E. Educação em saúde no Ensino Infantil: metodologias ativas na abordagem da ação extensionista. Rev enferm UFPE on line, Recife, v: 7 n: 1 p. 306-13, jan., 2013.
BRASIL. Ministério da Saúde. Secretaria de Atenção à Saúde. Departamento de Atenção Básica. Saúde na Escola. Brasília, 2009. 96 p.: il. - (Série B. Textos Básicos de Saúde) (Cadernos de Atenção Básica; n. 24).

BRASIL. Ministério da Saúde. Secretaria de Atenção à Saúde. Departamento de Ações Programáticas Estratégicas. Agenda de compromissos para a saúde integral da criança e redução da mortalidade infantil. Brasília, 2004. 80 p.: il.: color. - (Série A. Normas e Manuais Técnicos).

CRISTO, L. M. de O. de; ARAÚJO, T. C. C. F. de. Comunicação criança-acompanhante-pediatra: estudo observacional em diferentes níveis de assistência. Psicol. teor. prat., São Paulo, v. 17, n. 3, p. 89-103, dez. 2015.

ERDMANN, A. L.; SOUSA, F. G. M. de. Cuidando da criança na Atenção Básica de Saúde: atitudes dos profissionais da saúde. O Mundo da Saúde, São Paulo, v. 33, n. 2, p. 150-160, 20 mar. 2009.

GRABOIS, V.. Gestão do cuidado. In: GRABOIS, Victor; JÚNIOR, Walter Vieira Mendes; OLIVEIRA, Roberta Gondim de (Org.) Qualificação dos Gestores do SUS. 2. ed. Rio de Janeiro: Fiocruz/ENSP/EAD; 2011. p. 162-163. JÚNIOR, J. P. B. Fisioterapia e saúde coletiva: desafios e novas responsabilidades profissionais. Ciência \& Saúde Coletiva, v. 15, n. 1, P. 1627-1636, 2010.

LIMA, G. G. T.; SILVA, M. de F. de O. C.; COSTA, T. N. A.; NEVES, A. F. G. de B.; DANTAS, R. A.; LIMA, A. R. S. O. Registros Do Enfermeiro No Acompanhamento Do Crescimento E Desenvolvimento: Enfoque Na Consulta De Puericultura, Rev. Rene. Fortaleza, v. 10, n. 3, p. 117-124, jul./set. 2009.

NOVACZYK, A. B.; DIAS, N. da S.; GAIIVA, M. A. M. Atenção à saúde da criança na rede básica: análise de dissertações e teses de enfermagem. Rev. Eletr. Enf. v. 10, n. 4, P. 1124-37, 2008.

PANAZZOLO, P. R.; STOFFEL, M. O. S.; SIMON, R. A.; LIMA, M. C.; COSTANZI, C. B. Prevalência de sobrepeso e obesidade em escolares do município de Feliz, Rio Grande do Sul, Brasil, Revista Brasileira Medicina de Família Comunidade. Rio de Janeiro, v. 9, n. 31, p. 142-148, Abr/Jun. 2014.

PEREIRA, H. C.; LIMA, M. N. F. de A.; GARCIA, C. de L. Práticas educativas com mães, cuidadoras e crianças em situação de vulnerabilidade social. Revista Brasileira de Educação e Saúde, Pombal, v. 5, n. 2, p. 08-13, abr/jun. 2015.

ROCHA, A. C. D. Assistência pré-natal e vigilância do crescimento infantil no contexto da Estratégia Saúde da Família em Queimadas, Paraíba, Brasil. 2012, 121 f. Dissertação de Mestrado, UEPB, Campina Grande, 2012.

SÃO PAULO. SECRETARIA DE SAÚDE DO ESTADO DE SÃO PAULO. Linha de Cuidado da Criança. 
Orientações aos Gestores, aos Gerentes de Unidades de Saúde e Profissionais de Saúde dos diversos pontos de atenção. São Paulo, 2012.

VOLTA REDONDA. SECRETARIA MUNICIPAL DE SAÚDE DE VOLTA REDONDA. Saúde da Criança Área Técnica Programática da Saúde da Criança, Volta Redonda, 2015. Disponível em: <http://www.portalvr.com/saude/images/protocolo/Protoc olo \%20Saude \%20da\%20Criana\%20versao\%202015.pdf> Acesso em: 23 Set. 2016.
SILVA, N. D. S. H.; FILHO, F. L.; GAMA, M. E. Al.; LAMY, Z. de C.; PINHEIRO, A. do L.; SILVA, D.do N. Instrumentos de avaliação do desenvolvimento infantil de recém-nascidos prematuros. Rev. bras. crescimento desenvolv. hum., São Paulo, v. 21, n. 1, p. 85-98, 2011.

SILVA, N. C. B. da; NUNES, C. C.; BETTI, M. C. M.; RIOS, K. de S. A. Variáveis da família e seu impacto sobre o desenvolvimento infantil. Periódicos Eletrônicos de Psicologia (PePSIC), Ribeirão Preto, v. 16, n. 2, 2008. 